\title{
ROBUST RESIDUAL GENERATION FOR DIAGNOSIS INCLUDING A REFERENCE MODEL FOR RESIDUAL BEHAVIOR
}

\author{
Erik Frisk and Lars Nielsen
}

\author{
Dept. of Electrical Engineering, Linköping University \\ Linköping, Sweden \\ Email:frisk@isy.liu.se,lars@isy.liu.se
}

\begin{abstract}
The main goal when synthesizing robust residual generators, for diagnosis and supervision, is to attenuate influence from model uncertainty on the residual while keeping fault detection performance. In this paper, a design algorithm for robust residual generators is developed with two key elements. One is the use of a reference model that represents desired performance, i.e. time or frequency-domain specifications of fault response in the residual. The other is an optimization criterion, based on robust $\mathcal{H}_{\infty}$-filtering, used to synthesize the residual generator. Also, a methodology how to specify performance specifications to ensure good robustness properties of the optimal residual generator is developed.

Copyright (C) 1999 IFAC
\end{abstract}

Keywords: diagnosis, fault detection, robustness, reference model, uncertainty

\section{INTRODUCTION}

Diagnosis and supervision are important in many applications. Model based diagnosis uses a model to obtain residuals, which are signals that are zero in the fault free case and non-zero otherwise, to perform the diagnosis. Since available models of real processes always are uncertain, there is naturally a need for robust methods minimizing the sensitivity to the model uncertainties.

This paper addresses the problem of synthesizing and analyzing robust residual generators in the presence of parametric uncertainties and deterministic disturbances that influence the process. This problem is well studied and there exists many papers with different design focuses on the subject, e.g. (Frank and Ding, 1994; Mangoubi et al., 1995; Edelmayer et al., 1994; Mangoubi et al., 1994; Mangoubi et al., 1992; Eich and Oehler, 1997; Patton and Chen, 1993). Here, focus is on designing robust residual generators, dealing with model uncertainty, to fit in a structured residuals framework.

A main observation here is that, for this purpose, it is advantageous to introduce a reference model that describes desired behavior of the residuals with respect to faults. A theory is developed where the reference model idea and an optimization criterion are key elements.

\section{PROBLEM FORMULATION}

The system under consideration is assumed to be on the form

$$
y=G^{\Delta}(s) u+H^{\Delta}(s) d+L^{\Delta}(s) f
$$

where $y$ is the measurement vector, $u$ the control signal, $d$ is the disturbance vector, and $f$ is the fault vector. Matrices $G^{\Delta}(s), H^{\Delta}(s)$, and $L^{\Delta}(s)$ are all rational transfer matrices. The superscripts $\Delta$ indicate that the model is subject to bounded parametric uncertainties.

The residual generator is a finite dimensional linear filter $Q(s)$ that uses available known signals, i.e. $y$ and $u$, to form a vector of residuals, $r$, that can be used to detect and isolate the different faults $f$.

$$
r=Q(s)\left(\begin{array}{l}
y \\
u
\end{array}\right)
$$

The basic requirement on $Q(s)$, besides being $\mathcal{R} \mathcal{H}_{\infty}$, is that the residuals, $r$, should also be insensitive to control actions, $u$, and disturbances, $d$, but it should be sensitive to faults $f$.

The principle of fault isolation considered here is structured residuals where a subset of faults are decoupled in each residual. By generating a set of residuals where 
different subsets of faults are decoupled in each residual, fault isolation is possible.

$$
\begin{aligned}
& \text { Inserting (1) into (2) gives } \\
& r=Q(s)\left[\begin{array}{cc}
G^{\Delta}(s) & H^{\Delta}(s) \\
I & 0
\end{array}\right]\left(\begin{array}{l}
u \\
d
\end{array}\right)+Q(s)\left[\begin{array}{c}
L^{\Delta}(s) \\
0
\end{array}\right] f
\end{aligned}
$$

Nominally, to achieve decoupling of $u$ and $d$, the first term of (3) must be 0 while the second term must be $\neq 0$. However, with uncertain models it is in most cases impossible to get the first term $=0$ for all $\Delta$, i.e. for all possible instances of uncertainties, without loosing some or all of the desired fault sensitivity. Note that it is not always so; in some cases parametric uncertainty can be transformed into unknown input signals that can be decoupled with methods based on nominal models, see e.g. (Patton and Hou, 1998). However, generally some tradeoff between sensitivity to faults and disturbance/uncertainty attenuation is required.

The problem studied here is how to find the filter $Q(s)$ such that a proper trade off between fault sensitivity, disturbance attenuation and robustness towards model uncertainty is achieved. The solution is based on a performance specification in Section 3 and a synthesis procedure described in Section 4 based on robust $\mathcal{H}_{\infty}$ filtering.

\section{REFERENCE MODEL}

When synthesizing a robust residual generator, it is desired that the design freedom available should be used to achieve both robustness and detection performance, i.e. properties of the transfer function from faults to residuals. The question arises how this performance should be formulated. A natural choice is to introduce a reference model, $R(s)$, to describe the desired behavior of the residual vector $r$, with respect to faults, $f$. Define desired residual behavior, $r_{0}(s)$, of the residual, via the reference model as

$$
r_{0}=R(s) f(s)
$$

The matrix $R(s)$ is an arbitrary $\mathcal{R} \mathcal{H}_{\infty}$ transfer matrix of appropriate dimensions. The idea of a reference model has successfully been used to describe signal behavior in other fields like controller design (Åström and Wittenmark, 1984) and adaptive control (Åström and Wittenmark, 1989).

It is of course necessary that the reference model, $R(s)$, contains the necessary structure for $Q(s)$ to be a residual generator. This includes decoupling properties of faults, i.e. zeros at proper positions in $R(s)$ corresponding to the desired residual structure. The additional freedom in $R(s)$ can then be used to shape the desired frequency response in the residual from the faults.

Example 1. Suppose there are three modeled faults and it is desired to design a residual that:

(1) Decouples the first fault

(2) Responds to high frequency components $\left(\omega>\omega_{h}\right)$ of the second fault

(3) Responds to low frequency components $\left(\omega<\omega_{l}\right)$ of the third fault i.e. the residual should correspond to a row $\left[\begin{array}{lll}0 & 1 & 1\end{array}\right]$ in the residual structure. An example of $R(s)$ could then be

$$
R(s)=\left[\begin{array}{ll}
0 \frac{s}{s+\omega_{h}} & \frac{1}{s / \omega_{l}+1}
\end{array}\right]
$$

where $\omega_{h}$ and $\omega_{l}$ reflects the frequency ranges of interest.

Robust design (here) leads to an optimization over bounded parameter uncertainties where influence from worst-case disturbances and model uncertainties are minimized. This optimization problem may not be well formulated unless the reference model is attainable with a proper structure. Attainable reference model is here defined as

Definition 1. (Attainable Reference Model).

A reference model is said to be attainable if, with no model uncertainty, there exists a residual generator with the specified fault response.

If a non-attainable reference model is used, the synthesized residual generator may have unnecessary poor robustness properties which will be exemplified in Sections 5 and 8 .

\section{ROBUST RESIDUAL GENERATOR SYNTHESIS}

In any real-life system, perfect model knowledge can not be assumed, so influence on the residual from disturbances, control signal etc. can not be expected to be completely decoupled. The main idea with robust residual generation is to minimize these unwanted influences on the residuals while maintaining the fault sensitivity in the residuals. This trade-off, fault sensitivity vs. disturbance attenuation, is normally formulated as an optimization problem in different formulations and norms. A common choice is to utilize $\mathcal{H}_{\infty}$-theory to perform the synthesis. Powerful synthesis tools are important, but also worst-case analysis tools are important to aid e.g. robust threshold selection or robustness evaluation.

\subsection{Robustness Criterion}

The optimization criterion used here is formulated as a robust $\mathcal{H}_{\infty}$-filtering problem (Zhou et al., 1995), with an intuitive and appealing interpretation which is given after Eq. (5). The criterion is

$$
J=\sup _{v \in \mathcal{L}_{2}} \frac{\left\|r_{0}-r\right\|_{2}}{\|v\|_{2}}
$$

where $v=\left[\begin{array}{lll}u^{T} & f^{T} & d^{T}\end{array}\right]^{T}$. The optimization criterion $J$ is thus the worst case distance between the residual $r$ and the idealized residual $r_{0}$, defined by transfer matrix $R(s)$, normed by the size of the inputs. The optimal residual generator $Q(s)$ is the filter that minimizes $J$ for all $\|\Delta\|$ inside some bounded ball.

The optimization criterion $J$ can be rewritten as

$J=\sup _{v \in \mathcal{L}_{2}} \frac{\left\|r_{0}-r\right\|_{2}}{\|v\|_{2}}=\sup _{v \in \mathcal{L}_{2}} \frac{\left\|T_{z v}(s) v\right\|_{2}}{\|v\|_{2}}=\left\|T_{z v}(s)\right\|_{\infty}$ 
where $z(t)=r_{0}(t)-r(t)$, and

$$
T_{z v}(s)=\left[-G_{r u}^{\Delta}(s)\left(R(s)-G_{r f}^{\Delta}(s)\right)-G_{r d}^{\Delta}(s)\right]
$$

is the transfer matrix from $v(t)$ to $z(t)$. The transfer matrices from $u$ to $r, G_{r u}(s)$, from $d$ to $r, G_{r d}(s)$, and from $f$ to $r, G_{r f}(s)$ all depend on the residual generator $Q(s)$. Minimizing $J$, i.e. minimizing the $\infty$-norm of expression (5), has a simple interpretation, the first and third element makes sure that the influence from $u$ and $d$ on the residual are attenuated. The middle term keeps fault sensitivity, and also shapes the fault to residual transfer function $G_{r f}(s)$ by minimizing the distance to the reference model $R(s)$.

The optimization/performance index minimizes the $a b$ solute difference between $R(s)$ and $G_{r f}^{\Delta}(s)$. A reasonable assumption is that it is the relative difference that need to be minimized, otherwise in high-gain models even very small relative errors will dominate the loss function and therefore move away optimization focus from robustness to fault sensitivity in an unwanted manner. Therefore it is important to normalize and weigh the model appropriately to avoid such effects.

Remark 1 It is obvious that the optimal value of $J$ also delivers constraints on the size (norm) of $r$ in the fault-free and faulty case which can be used to guide threshold selection.

Remark 2 A special case of Eq. (4) is treated in (Niemann and Stoustrup, 1997), where integrated residual generator and controller design is presented. The optimization criterion associated with the residual generator design is closely related with the criterion presented here. By setting $r_{0}(t)=f(t)$, i.e. strive for a diagonal residual structure with unit gain from fault $i$ to residual $i$, the optimization criteria become the same.

\subsection{Computational Framework}

The residual generation optimization problem can be described by an upper and lower LFT, including the structured parametric uncertainty, as in Figure 1, where $P(s)$ is an augmented system description including a description on how the parametric uncertainties $\Delta$ influence the system, fault models, disturbance models, dynamic weighting matrices and also the reference model.

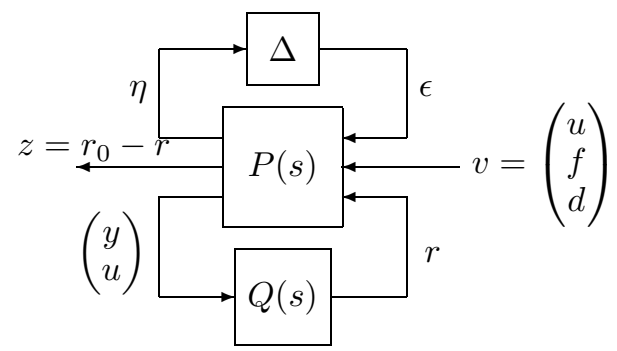

Fig. 1. LFT formulation of residual generation problem
With this problem setup, there exist algorithms minimizing $J$ with respect to $Q(s)$ by e.g. $\mu$-synthesis. The algorithm used in this work is basic DK-iterations which, although heuristic and with no convergence guarantees, have in practice shown reliable performance (Balas et al., 1993).

\section{BACKGROUND EXAMPLE ON REFERENCE MODEL DESIGN}

The idea of a reference model for residual behavior has been introduced, and was in the previous section used in the criterion for robust design. Before going into details of the design of the reference model, a simple stylized static example will illustrate the necessity of using a well formulated reference model. The discussion forms a background and leads to the detailed design in Section 6.

Example 2. Consider the static system

$$
y_{1}=\left(1+\delta_{1}\right) x+3 f_{1} \quad y_{2}=\left(2+\delta_{2}\right) x+4 f_{2}
$$

where $x$ is an unknown disturbance and $|\delta|<\frac{1}{2}$. Suppose it is desired that the disturbance $x$ does not influence the residual while $f_{1}$ and $f_{2}$ has significant influence which is the same as saying that $r$ should respond to a row [1 1 ] in a residual structure.

The residual generator is formed as

$$
r=q_{1} y_{1}+q_{2} y_{2}
$$

First attempt A naive choice of $R(s)$ without any further thought might be to aim for unit gain from both faults, i.e. $R(s)=\left[\begin{array}{ll}1 & 1\end{array}\right]$. Performing a design as described in the previous section and denoting the resulting residual generator with $q_{a}=\left[q_{1} q_{2}\right]^{T}$. Robustness is evaluated by determining the worst-case gain from the disturbance to the residual. This corresponds to measuring the size of the third term in (5). Since there is no control signal in this example, this measure indicates performance of the filter in a no fault situation.

$\max _{|\delta|<\frac{1}{2}}\left\|k_{r x}\right\|=\max _{|\delta|<\frac{1}{2}}\left\|\left[\left(1+\delta_{1}\right) \quad\left(2+\delta_{2}\right)\right] q_{a}\right\|=1.683$

That is, the worst-case gain from $x$ to $r$ is $k_{r x}=1.683$.

Second attempt A little more thought on the problem leads to a second attempt based on a nominal design. If no uncertainty is present, then $\delta=0$ and a natural choice of $q$ would satisfy

$$
q_{2}=-\frac{1}{2} q_{1}
$$

to completely decouple the disturbance $x$. The relation between the gain from $f_{1}$ to $r, k_{r f_{1}}$, and $f_{2}$ to $r, k_{r f_{2}}$, is then related by

$$
\frac{k_{f_{1}}}{k_{r_{2}}}=\frac{3 q_{1}}{4 q_{2}}=-\frac{2 \cdot 3}{1 \cdot 4}=-\frac{3}{2}
$$

Using this nominal information will significantly improve robustness properties of the design. Let the ele- 
ments in $R(s)$ be proportional to nominal gains from the faults to the residual $k_{r f_{1}}$ and $k_{r f_{2}}$, e.g.

$$
R(s)=\left[-\frac{3}{2} 1\right]
$$

Note that this choice of $R(s)$ also corresponds to a [1 1 1] residual structure. A new design with this $R(s)$ results in a $q_{b}$, normed to be of the same size as $q_{a}$, in the sense that $\left|q_{a}\right|=\left|q_{b}\right|$. This is important to enable a fair robustness comparison. The second design has robustness properties

$$
\max _{|\delta|<\frac{1}{2}}\left\|\left[\left(1+\delta_{1}\right) \quad\left(2+\delta_{2}\right)\right] q_{b}\right\|=0.7906
$$

Comparison Thus, a significant improvement on robustness properties was achieved in the second design compared to the first design. This is because in the first design, $R(s)$ reflected unrealistic performance specifications that inflicted unnecessary poor robustness properties on the residual generator. This issue will be further explored below and in the concluding example in Section 8.

Remark 1 It is worth noting that even in a no uncertainty case with $\delta_{1}=\delta_{2}=0$, the first design attempt would not have found a solution where the unknown disturbance $x$ is completely decoupled, even though such a solution exists. The reason for this, which is the same reason that made the second solution more robust, is that during optimization, freedom is spent to make $G_{r f}(s)-R(s)$ as small as possible. If an unrealistic $R(s)$ is used, freedom is spent on an impossible task that often is of no importance to the FDI problem. A suitable choice of $R(s)$ helps focusing on the robustness properties while keeping fault sensitivity and conforming to the specified performance.

\section{FORMING THE REFERENCE MODEL}

From the example above it is clear that a poorly chosen reference model, i.e. a reference model with unrealistic performance properties, can result in a residual generator with unnecessary poor robustness properties. The main idea is thus to use a nominal design of the residual generator to shape the reference model when synthesizing the robust residual generator, thus assuring attainable reference models. This is to avoid specifying an unrealistic performance criterion and thereby inflicting unnecessary poor robustness properties on the residual generator.

The formation of the criterion for the robust design is straightforward, given that a nominal residual generator, i.e. a $Q_{\text {nom }}(s)$, has been derived that nominally fulfills all demands. The reference model $R(s)$ is then selected as

$$
R(s)=Q_{n o m}(s)\left[\begin{array}{c}
L(s) \\
0
\end{array}\right]
$$

since this is the nominal fault to residual transfer function, compare with Eq. (3).

Of course, if no design based on a nominal model is available that meets the requirements of the application, then no feasible design with an uncertain model is available either.

\subsection{Nominal Design}

It is clear that the residual generator design problem is, in a structured residuals framework, essentially a decoupling problem which can be solved by a number of known methods. Freedom available during a nominal design are (1) the poles, i.e. the denominator, of the residual generator that can be selected arbitrarily and (2) freedom when selecting the numerator of the residual generator. Issue (2) means that there might exist several numerators of the residual generator that achieves the desired decoupling and different numerators may have different robustness and fault sensitivity properties. In an observer formulation of the residual generator this corresponds to selecting different observer modes.

Remark It is possible that, even with nominal models, no disturbance decoupling residual generator exists that conforms to the desired residual structure. Then a residual generator can be synthesized where disturbances and faults (according to the residual structure) are approximately decoupled. This is often stated as an optimization problem and solved in different ways by many different methods, e.g. by $\mathcal{H}_{\infty}$ methods (Frank and Ding, 1994; Qiu and Gertler, 1994), singular value truncation (Lou et al., 1986) and other methods (Wünnenberg, 1990). If the resulting residual generator, which doesn't perfectly decouple disturbances, still complies with the requirements on the diagnosis system, the nominal residual generator can be used to form a reference model according to (7) and robust synthesis following this paper can be performed.

\section{Example of reference model design}

Example 3. Assume a 1-input, 2-output, second order process with sensor and actuator faults:

$$
y=\left[\begin{array}{c}
\frac{\beta}{s+\alpha} \\
\frac{\beta}{s(s+\alpha)}
\end{array}\right]\left(u+f_{3}\right)+\left(\begin{array}{l}
f_{1} \\
f_{2}
\end{array}\right)
$$

If a residual with structure $\left[\begin{array}{lll}1 & 0 & 1\end{array}\right]$ is desired, then the only possible residual generator $Q_{n o m}(s)$ (apart from the poles and any post filtering) is

$$
Q_{n o m}(s)=\frac{\gamma}{s+\gamma}[s+\alpha 0-\beta]
$$

The reference model $R(s)$ corresponding to the nominal design is then

$$
R(s)=Q_{\text {nom }}(s)\left[\begin{array}{c}
L(s) \\
0
\end{array}\right]=\frac{\gamma}{s+\gamma}\left[\begin{array}{lll}
s+\alpha & 0 & \beta
\end{array}\right]
$$

\subsection{Discussion of Design Choices}

When choosing $Q_{n o m}(s)$, and thereby the reference model $R(s)$, two degrees of freedom have to be chosen by the designer (here it is assumed that in the first-step 
nominal design, perfectly decoupling residual generators exist):

- The numerator may need to be chosen, especially since the space of decoupling numerators can have dimensionality greater than one, which means that there are several numerators to choose from.

- The denominator need to be chosen. The dynamics (poles) of the residual generator is completely free, conditioned that the degree is greater or equal to the numerator degree.

These choices are then held fix during optimization. Instead of specifying the poles individually, they could of course be obtained almost automatically using a band-width requirement together with a Butterworth structure (Åström and Wittenmark, 1984).

It might be argued that by fixating these two degrees of freedom limits the freedom in the optimization to achieve robustness against parametric variations and that these two variables should be optimized over. It is well known that in nominal design of residual generators, the poles of the residual generator can be chosen arbitrarily. Therefore, for any fixed set of uncertain parameters $\Delta$ would only influence the numerator of the residual generator. Therefore, it is believed that fixating the poles does not severely influence the robustness properties of the optimal residual generator.

\section{SUMMARY OF DESIGN METHOD}

An advantage with the problem formulation used here is that it is possible to incorporate desired fault to residual structure and performance in the optimization criterion, i.e. the reference model.

Recall the optimization criterion

$$
\begin{aligned}
J & =\sup _{v \in \mathcal{L}_{2}} \frac{\left\|r_{0}-r\right\|_{2}}{\|v\|_{2}}=\left\|T_{z v}(s)\right\|_{\infty}= \\
& =\left\|\left[-G_{r u}^{\Delta}(s)\left[R(s)-G_{r f}^{\Delta}(s)\right]-G_{r d}^{\Delta}(s)\right]\right\|_{\infty}
\end{aligned}
$$

where $G_{r u}^{\Delta}(s)$ is the transfer function from $u$ to $r$, $G_{r f}^{\Delta}(s)$ is the transfer function from $f$ to $r$, and $G_{r d}^{\Delta}(s)$ is the transfer function from $d$ to $r$. These are given directly by Eq. (3) and they are all dependent on the residual generator $Q(s)$. The optimization goal is then to find the $Q(s)$ that minimizes $J$ under bounded parametric uncertainties $\Delta$. The first and third components of $J$ then decouples $u$ and $d$ and the second shapes the residual response of the faults.

The synthesis procedure is as follows

(1) Design the reference model according to (7) by performing a nominal design $Q_{n o m}(s)$.

(2) Introduce weighting matrices to focus on important frequency ranges

(3) Optimize (minimize) $J$ under structured uncertainties $\Delta$ with $\mu$-synthesis.

(4) If needed, apply model reduction techniques on the resulting residual generator and re-evaluate robust performance via $\mu$-analysis.

The main designer interaction is in step 1 where the nominal design, and thereby the reference model, is designed, and in step 2 where knowledge of the process or demands on the diagnosis system is used to shape the optimization criterion. Design choices during step 1 were discussed in Section 6.2.

\section{ILLUSTRATIVE DYNAMIC EXAMPLE}

In this section, the concepts introduced in the paper are applied to a small dynamic example with parametric uncertainties. Three designs are made, one nominal, one with an ad-hoc reference model and one design with a reference model based on the nominal design. Robustness properties of the three designs are then compared.

\subsection{Model}

Reconsider Example 3, inspired from a second order DC-servo model. Assume uncertainties in moment of inertia, modeled by $\delta_{1}$, and in the viscous friction, modeled by $\delta_{2}$. The model can then be written on statespace form as

$$
\begin{aligned}
\left(1+\delta_{1}\right) \dot{x}_{1} & =-\left(\alpha+\delta_{2}\right) \dot{x}_{1}+\beta\left(u+f_{3}\right) \\
\dot{x}_{2} & =x_{1} \\
y_{1} & =x_{1}+f_{1} \\
y_{2} & =x_{2}+f_{2}
\end{aligned}
$$

\subsection{Residual Generator Specifications}

Suppose a residual generator is to be synthesized to conform to a [ $\left[\begin{array}{lll}1 & 0 & 1\end{array}\right]$ structure, i.e. decouple sensor fault 2 while keeping sensitivity to the other two faults. The parameters are $\alpha=1$ and $\beta=4$. Three different designs are considered:

- The nominal design is made as in Example 3. The nominal residual generator $Q_{n o m}(s)$ was given by (8).

- The robust residual generator with a non-attainable $R(s)=\left[\begin{array}{lll}1 & 0 & 1\end{array}\right]$ is synthesized using the method in Section 7. The resulting residual generator is denoted $Q_{n a}(s)$. This choice of $R(s)$ is related to the first naive attempt in Example 2.

- A robust design with an attainable $R(s)$, based on the nominal design $Q_{n o m}(s)$, is performed using the method in Section 7 and is denoted $Q_{a t t}(s)$.

\subsection{Robustness Comparison}

It is hard to make a fair comparison of robustness because of the many different definitions of robust residual generators. Here, the designs have been normed to have equal static fault-to-residual gains (the 2-norm of $G_{r f}(0)$ is equal for all designs). Then, the robustness comparison measure used is how large $G_{r u}(s)$ gets under bounded parametric uncertainties in a worst-case situation. This is the same robustness evaluation criterion as in the static Example 2 and corresponds to the operation of the residual generators in a fault-free situation. 
Robustness comparison is performed with a theorem from robust control on robust performance. Let $\mathcal{F}_{u}(P, \Delta)$ be an upper LFT of $P$ with respect to $\Delta$.

Theorem 1. (Robust Performance). Let $\beta>0$. For all $\|\Delta(s)\|_{\infty}<\frac{1}{\beta}, \mathcal{F}_{u}(P, \Delta)$ is well-posed, internally stable and $\left\|\mathcal{F}_{u}(P, \Delta)\right\|_{\infty} \leq \beta$ if and only if

$$
\sup _{\omega \in \mathbb{R}} \mu_{\Delta_{P}}(P(j \omega)) \leq \beta
$$

where

$$
\Delta_{P}=\left\{\left[\begin{array}{cc}
\Delta & 0 \\
0 & \Delta_{f}
\end{array}\right]: \Delta \in \boldsymbol{\Delta}, \Delta_{f} \in \mathbb{C}^{q_{2} \times q_{2}}\right\}
$$

Figure 2 shows $\mu_{\Delta_{P}}\left(G_{r u}(j \omega)\right)$ for the three designs. The interpretation of the plot is, according to the theorem above, when $\mu_{\Delta_{P}}\left(G_{r u}(j \omega)\right)<\beta$ then for all $\|\Delta\|_{\infty}<1 / \beta$ it holds that $\left\|G_{r u}^{\Delta}(s)\right\|<\beta$. So the smaller $\beta$, i.e. peak-value of the $\mu$-plot, the more robust is the design. The plot gives that $Q_{n o m}(s), Q_{n a}(s)$,

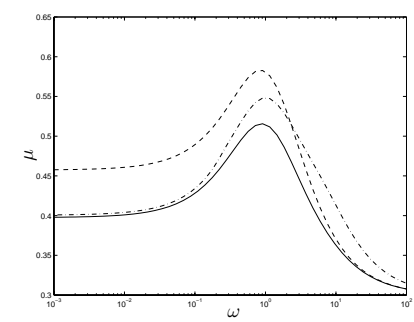

Fig. 2. Plot of $\mu_{\Delta_{P}}\left(G_{r u}(j \omega)\right)$ to evaluate robustness properties. The dashed, dash-dotted, and the solid lines corresponds to $Q_{n o m}(s), Q_{n a}(s)$ and $Q_{\text {att }}(s)$ respectively.

and $Q_{\text {att }}$ gives $\beta=0.6,0.55,0.5$ respectively. Thus, $Q_{n a}(s)$ suffers from up to $15 \%$ unnecessary loss of robustness compared to $Q_{a t t}(s)$. In fact, $Q_{n a}(s)$ is even worse than the nominal design for large frequencies. This means that a robust design not considering a proper reference model can in fact be less robust than a nominal design.

This example, and the example in Section 5, show that a significant increase in robustness was achieved by robust design with a reference model chosen via a nominal design.

\section{CONCLUSIONS}

A theory for robust residual design has been developed where a key element is the use of a reference model. The reference model represents desired structure and performance of the synthesized residual generator. It is a condensed formulation including structural requirements on fault decoupling, to make the synthesized residual generator fit in a larger diagnosis system based on structured residuals. It also includes performance issues such as fault response in the residual. Without considering structural constraints, it is possible to form unrealistic performance demands and it is shown by examples how this can de-emphasize the robustness parts of the optimization and lead to a design with unnecessary poor robustness properties. A methodology how to select realistic reference models is presented where the design freedom available is explicit and intuitive. The optimization algorithms used to synthesize the residual generator rely on established and efficient methods. The designer of a diagnosis system is thus provided with a tool where it is easy to specify desired behavior without violating structural requirements.

\section{REFERENCES}

Åström and Wittenmark (1984). Computer Controlled Systems - Theory and Design. Prentice Hall.

Åström and Wittenmark (1989). Adaptive Control. Prentice Hall.

Balas, G.J., J.C. Doyle, K. Glover, A. Packard and R. Smith (1993). $\mu$-Analysis and Synthesis Toolbox. Math Works Inc.

Edelmayer, A., J. Bokor and L. Keviczky (1994). An $H_{\infty}$ filtering approach to robust detection of failures in dynamic systems. In: Proceedings Decision \& Control. pp. 3037-3039.

Eich, J. and R. Oehler (1997). On the application of the generalized structured singular value to robust fdi system design. In: IFAC Fault Detection, Supervision and Safety for Technical Processes. Hull, UK. pp. 885-890.

Frank, P.M. and X. Ding (1994). Frequency domain approach to optimally robust residual generation and evaluation for model-based fault diagnosis. Automatica 30(5), 789-804.

Lou, X., A. Willsky and G. Verghese (1986). Optimally robust redundancy relations for failure detection in uncertain systems. Automatica 22(3), 333-344.

Mangoubi, R., B.D. Appleby and G.C. Verghese (1994). Stochastic interpretation of $H_{\infty}$ and robust estimation. In: Proceedings Decision \& Control. pp. 3943-3948.

Mangoubi, R., B.D. Appleby and J. Farrell (1992). Robust estimation in fault detection. In: Proceedings Decision \& Control. pp. 2317-2322.

Mangoubi, R., B.D. Appleby, G.C. Verghese and W.E. VanderVelde (1995). A robust failure detection and isolation algorithm. In: Proceedings Decision \& Control. pp. 2377-2382.

Niemann, H. and J. Stoustrup (1997). Integration of control and fault detection: nominal and robust design. IFAC Fault Detection, Supervision and Safety for Technical Processes. Hull, UK. pp. 341346.

Patton, R.J. and J. Chen (1993). Optimal unknown input distribution matrix selection in robust fault diagnosis. Automatica 29(4), 837-841.

Patton, R.J. and M. Hou (1998). Design of fault detection and isolation observers: A matrix pencil approach. Automatica.

Qiu, Z. and J. Gertler (1994). Robust FDI systems and $H_{\infty}$-optimization. IFAC Fault Detection, Supervision and Safety for Technical Processes. Espoo, Finland. pp. 247-252.

Wünnenberg, Jürgen (1990). Observer-Based Fault Detection in Dynamic Systems. PhD thesis. University of Duisburg.

Zhou, K., J.C. Doyle and K. Glover (1995). Robust and Optimal Control. Prentice Hall. 